\title{
Bohr Hamiltonian with a deformation-dependent mass term: physical meaning of the free parameter
}

\author{
Dennis Bonatsos ${ }^{1}, \mathrm{~N}$ Minkov $^{2}$ and D Petrellis ${ }^{3}$ \\ ${ }^{1}$ Institute of Nuclear and Particle Physics, National Center for Scientific Research \\ "Demokritos", GR-15310 Aghia Paraskevi, Attiki, Greece \\ ${ }^{2}$ Institute of Nuclear Research and Nuclear Energy, Bulgarian Academy of Sciences, \\ 72 Tzarigrad Road, 1784 Sofia, Bulgaria \\ ${ }^{3}$ Department of Physics, University of Istanbul, 34134 Vezneciler, Istanbul, Turkey \\ E-mail: bonat@inp.demokritos.gr, nminkov@inrne.bas.bg, \\ dimitrios.petrellis@istanbul.edu.tr
}

\begin{abstract}
Embedding of the 5-dimensional (5D) space of the Bohr Hamiltonian with a deformation-dependent mass (DDM) into a 6-dimensional (6D) space shows that the free parameter in the dependence of the mass on the deformation is connected to the curvature of the $5 \mathrm{D}$ space, with the special case of constant mass corresponding to a flat 5D space. Comparison of the DDM Bohr Hamiltonian to the 5D classical limit of Hamiltonians of the 6D interacting boson model (IBM), shows that the DDM parameter is proportional to the strength of the pairing interaction in the $\mathrm{U}(5)$ (vibrational) symmetry limit, while it is proportional to the quadrupole-quadrupole interaction in the $\mathrm{SU}(3)$ (rotational) symmetry limit, and to the difference of the pairing interactions among $s, d$ bosons and $d$ bosons alone in the $\mathrm{O}(6)(\gamma$-soft) limit. The presence of these interactions leads to a curved 5D space in the classical limit of IBM, in contrast to the flat 5D space of the original Bohr Hamiltonian, which is made curved by the introduction of the deformation-dependent mass.
\end{abstract}

Keywords: Bohr collective model, Interacting Boson Model, deformation-dependent mass

PACS numbers: 21.60.Ev, 21.60.Fw 


\section{Introduction}

The advent of supersymmetric quantum mechanics (SUSYQM) [1, 2] has led to exact solutions of Hamiltonians with position-dependent masses (PDM) [3, 4]. Positiondependent effective masses have been widely used [3, 4] in compositionally graded crystals, semiconductor theory, quantum dots, quantum liquids, liquid crystals and metal clusters. In this paper we consider the use of a position-dependent effective mass in the structure of atomic nuclei, showing that this is not a mathematical trick introducing an extra parameter, but a necessity arising from concrete physical requirements.

Collective effects in atomic nuclei are described in two alternative frameworks, the collective model of Bohr and Mottelson [5, 6] and the interacting boson model (IBM) of Arima and Iachello [7]. A major drawback of the collective model of Bohr and Mottelson has been over the years the rapid increase of the nuclear moments of inertia as a function of the nuclear deformation [8]. A possible solution to this problem has been suggested [9] recently by allowing the nuclear mass to depend on the deformation, taking advantage of techniques developed for Hamiltonians with masses dependent on the coordinates [3, 4], with exact analytical solutions obtained for certain classes of potentials through the use of supersymmetric quantum mechanics (SUSYQM) [1, 2]. Such a solution has been obtained [9] for the deformation-dependent mass (DDM) Bohr Hamiltonian [5] with a Davidson potential [10], in which the mass depends on the deformation through a specific function containing a free parameter, $a$. It is the purpose of the present work to examine the physical relevance of this free parameter. This will be achieved by embedding [11] the 5-dimensional (5D) space of the Bohr Hamiltonian into a 6-dimensional (6D) space, thus connecting the DDM free parameter to the curvature of the 5D space, a procedure of general applicability in Hamiltonians using position-dependent masses.

On the other hand, the algebraic interacting boson model (IBM) [7], which is known to possess an overall $\mathrm{U}(6)$ symmetry in a $6 \mathrm{D}$ space, is also known to have a classical limit [12, 13, 14, 15, corresponding to a 5D space. Comparison of the DDM Bohr Hamiltonian to the classical limit of the most general IBM Hamiltonian, as well as to the special cases 
corresponding to the three limiting symmetries [U(5) (vibrational), SU(3)(rotational) and $\mathrm{O}(6)(\gamma$-unstable $)]$ of the IBM, results in connecting the DDM free parameter to certain IBM parameters, thus attributing to it a specific physical meaning.

Before proceeding, two comments are in place.

1) The need for different mass parameters in the various low-lying nuclear collective bands, and even for a mass tensor within the Bohr Hamlitonian, has been pointed out by Jolos and von Brentano [16, 17, 18]. This method has been recently extended to the description of odd nuclei [19, 20].

2) The Davidson potential has also been used in the framework of the algebraic collective model [21, 22, 23], which allows rapidly converging numerical calculations for nuclei of any shape.

The DDM Bohr Hamiltonian will be briefly presented in Section 2, while in Section 3 its embedding into a $6 \mathrm{D}$ space will be developed. The classical limit of the IBM Hamiltonian will be compared to the DDM Bohr Hamiltonian in Section 4, while in Section 5 the results will be discussed.

\section{The DDM Bohr Hamiltonian}

The original Bohr Hamiltonian [5] is

$$
\begin{gathered}
H_{B}=-\frac{\hbar^{2}}{2 B}\left[\frac{1}{\beta^{4}} \frac{\partial}{\partial \beta} \beta^{4} \frac{\partial}{\partial \beta}+\frac{1}{\beta^{2} \sin 3 \gamma} \frac{\partial}{\partial \gamma} \sin 3 \gamma \frac{\partial}{\partial \gamma}\right. \\
\left.-\frac{1}{4 \beta^{2}} \sum_{k=1,2,3} \frac{Q_{k}^{2}}{\sin ^{2}\left(\gamma-\frac{2}{3} \pi k\right)}\right]+V(\beta, \gamma),
\end{gathered}
$$

where $\beta$ and $\gamma$ are the usual collective coordinates $(\beta$ being a deformation coordinate measuring departure from spherical shape, and $\gamma$ being an angle measuring departure from axial symmetry), while $Q_{k}(k=1,2,3)$ are the components of angular momentum

in the intrinsic frame, and $B$ is the mass parameter, which is usually considered as being a constant. 
Bohr Hamiltonian with a deformation-dependent mass term

In the DDM case, the mass is assumed to be a function of the deformation

$$
B(\beta)=\frac{B_{0}}{(f(\beta))^{2}},
$$

where $B_{0}$ is a constant.

The Schrödinger equation for the Bohr Hamiltonian $H$ with deformation-dependent mass (DDM) has the form [9]

$$
\begin{gathered}
H \Psi=\left[-\frac{1}{2} \frac{\sqrt{f}}{\beta^{4}} \frac{\partial}{\partial \beta} \beta^{4} f \frac{\partial}{\partial \beta} \sqrt{f}-\frac{f^{2}}{2 \beta^{2} \sin 3 \gamma} \frac{\partial}{\partial \gamma} \sin 3 \gamma \frac{\partial}{\partial \gamma}\right. \\
\left.+\frac{f^{2}}{8 \beta^{2}} \sum_{k=1,2,3} \frac{Q_{k}^{2}}{\sin ^{2}\left(\gamma-\frac{2}{3} \pi k\right)}+v_{e f f}\right] \Psi=\epsilon \Psi,
\end{gathered}
$$

where reduced energies $\epsilon=B_{0} E / \hbar^{2}$ and reduced potentials $v=B_{0} V / \hbar^{2}$ have been used, the effective potential being

$$
v_{e f f}=v(\beta, \gamma)+\frac{1}{4}(1-\delta-\lambda) f \nabla^{2} f+\frac{1}{2}\left(\frac{1}{2}-\delta\right)\left(\frac{1}{2}-\lambda\right)(\nabla f)^{2},
$$

where $\delta$ and $\lambda$ are free parameters, further discussed in Ref. 9].

In order to achieve exact separation of variables [24, 25], potentials of the form

$$
v(\beta, \gamma)=u(\beta)+\frac{f^{2}}{\beta^{2}} w(\gamma)
$$

have been considered. In the place of $u(\beta)$, the Davidson [10] and Kratzer [26] potentials have been used [9, 27]. For $\gamma$-unstable nuclei one has $w(\gamma)=0$, while for prolate deformed nuclei a harmonic oscillator potential centered around $\gamma=0$ is used, and for triaxial nuclei a harmonic oscillator potential centered around $\gamma=\pi / 6$ is used. The potential $w(\gamma)$ is playing no role in what follows, since it always appears within the effective potential $v_{e f f}$, which contains no derivatives with respect to the variables $\beta$ and $\gamma$. It should be noticed that for both the Davidson and the Kratzer potentials, the "radial" equation (the equation involving the variable $\beta$ ) is acquiring a common form [9, 27] for all three different cases of $w(\gamma)$ mentioned above.

In the case of the Davidson potential [10]

$$
u(\beta)=\beta^{2}+\frac{\beta_{0}^{4}}{\beta^{2}},
$$


where $\beta_{0}$ is a parameter indicating the position of the minimum of the potential, the deformation function has the special form [9]

$$
f(\beta)=1+a \beta^{2}, \quad a \ll 1,
$$

where $a$ is a free parameter. It is the physical content of this parameter which will be considered in the present study.

Performing the derivation in the first term of Eq. (3), the Hamiltonian in the case of the Davidson potential takes the form

$$
\begin{aligned}
H \Psi=\left[-\frac{2}{\beta}\left(1+a \beta^{2}\right)\left(1+2 a \beta^{2}\right) \frac{\partial}{\partial \beta}-\frac{1}{2}\left(1+a \beta^{2}\right)^{2} \frac{\partial^{2}}{\partial \beta^{2}}-\frac{\left(1+a \beta^{2}\right)^{2}}{2 \beta^{2} \sin 3 \gamma} \frac{\partial}{\partial \gamma} \sin 3 \gamma \frac{\partial}{\partial \gamma}\right. \\
\left.+\frac{\left(1+a \beta^{2}\right)^{2}}{8 \beta^{2}} \sum_{k=1,2,3} \frac{Q_{k}^{2}}{\sin ^{2}\left(\gamma-\frac{2}{3} \pi k\right)}+v_{e f f}^{\prime}\right] \Psi=\epsilon \Psi,
\end{aligned}
$$

where

$$
v_{e f f}^{\prime}=v_{e f f}-\left(\frac{5}{2} a+3 a^{2} \beta^{2}\right) .
$$

This result will be used in Section 4 for comparison to the classical limit of the most general IBM Hamiltonian.

\section{Embedding of the DDM Bohr Hamiltonian}

\subsection{Embedding from two into three dimensions}

In order to clarify the notions of curved space, embedding into higher dimensions, and conformal factor, we start with some simple considerations.

The surface of a sphere is a 2D (two-dimensional) curved space of constant curvature, on which the Pythagorean theorem is not valid. The Pythagorean theorem is

restored if we add one more dimension, going to the familiar 3D space, i.e., embedding the $2 \mathrm{D}$ sphere in a $(2+1) \mathrm{D}$ space, in which the sphere is described by the constraint

$$
x_{1}^{2}+x_{2}^{2}+x_{3}^{2}=R^{2}
$$

where $R$ is the radius of the sphere, while the length element in cartesian coordinates is

$$
d l^{2}=d x_{1}^{2}+d x_{2}^{2}+d x_{3}^{2}
$$


Bohr Hamiltonian with a deformation-dependent mass term

Using Eq. (10) we can eliminate the 3rd coordinate, obtaining

$$
d l^{2}=d x_{1}^{2}+d x_{2}^{2}+\frac{\left(x_{1} d x_{1}+x_{2} d x_{2}\right)^{2}}{R^{2}-x_{1}^{2}-x_{2}^{2}} .
$$

Replacing the cartesian coordinates of the $2 \mathrm{D}$ euclidean space by polar ones

$$
x_{1}=r \cos \theta, \quad x_{2}=r \sin \theta,
$$

this can be rewritten as

$$
d l^{2}=d r^{2}\left(1-\frac{r^{2}}{R^{2}}\right)^{-1}+r^{2} d \theta^{2} .
$$

Performing the conformal transformation (i.e., a transformation not affecting the angles, but only modifying the radial coordinate)

$$
r=r_{1}\left(1+\frac{r_{1}^{2}}{4 R^{2}}\right)^{-1}
$$

the length element takes the form

$$
d l^{2}=\left(1+\frac{r_{1}^{2}}{4 R^{2}}\right)^{-2}\left(d r_{1}^{2}+r_{1}^{2} d \theta^{2}\right)
$$

which is proportional to the euclidean expression

$$
\bar{d} l^{2}=d r_{1}^{2}+r_{1}^{2} d \theta^{2}
$$

differing only by the conformal factor in front of it in Eq. 16.

Following the same steps in the embedding from three into four dimensions [28, 29] and using spherical coordinates one obtains

$$
d l^{2}=\left(1+\frac{r_{1}^{2}}{4 R^{2}}\right)^{-2}\left(d r_{1}^{2}+r_{1}^{2}\left(d \theta^{2}+\sin ^{2} \theta d \phi^{2}\right)\right)
$$

which is proportional to the euclidean expression

$$
\overline{d l}^{2}=d r_{1}^{2}+r_{1}^{2}\left(d \theta^{2}+\sin ^{2} \theta d \phi^{2}\right)
$$

\subsection{Stereographic projection}

Through a stereographic projection a point $\left(x_{1}, x_{2}, x_{3}\right)$ on the surface of a sphere is

projected onto a point $\left(X_{1}, X_{2}\right)$ on the plane passing through the south pole of the sphere and being perpendicular to the axis connecting the poles of the sphere. The projection is made through a line starting from the north pole of the sphere, passing 
through the $\left(x_{1}, x_{2}, x_{3}\right)$ point and ending at the $\left(X_{1}, X_{2}\right)$ point. This is schematically illustrated in Fig. 1, where the plane formed by the axes 2 and 3 is depicted.

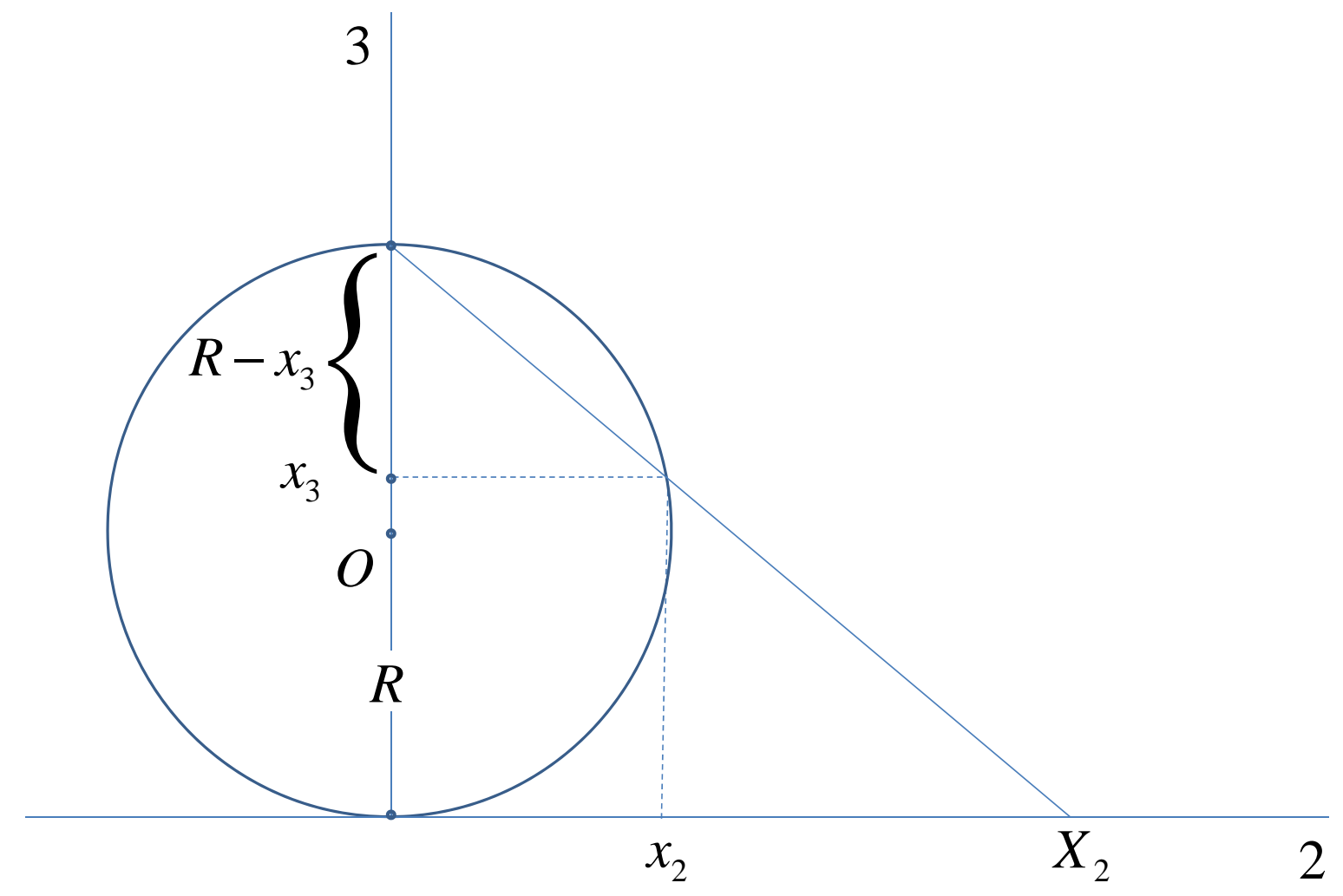

Figure 1. (Color online) Schematic illustration (two-dimensional intersection) of the stereographic projection of a point $\left(x_{1}, x_{2}, x_{3}\right)$ on the surface of a sphere of radius $R$ onto a point $\left(X_{1}, X_{2}\right)$ on a plane passing through the south pole of the sphere and being perpendicular to the axis connecting the poles of the sphere. The intersection of the sphere with the plane formed by the axes 2 and 3 is shown. The three axes are labeled by $1,2,3$, while distances on them are measured starting from the center of the sphere, $O$. The similarity between the two triangles depicted, leads directly to the second equation in 20 .

If $R$ is the radius of the sphere, and the north pole is located on the positive $x_{3}$ axis, from simple geometrical considerations one sees from Fig. 1 that

$$
\frac{x_{1}}{R-x_{3}}=\frac{X_{1}}{2 R}, \quad \frac{x_{2}}{R-x_{3}}=\frac{X_{2}}{2 R} .
$$


Solving the system of these two equations plus Eq. 10 for $x_{1}, x_{2}, x_{3}$ we find

$$
x_{1}=\frac{X_{1}}{D}, \quad x_{2}=\frac{X_{2}}{D}, \quad x_{3}=\frac{R}{D}\left(-1+\frac{X_{1}^{2}+X_{2}^{2}}{4 R^{2}}\right), \quad D=1+\frac{X_{1}^{2}+X_{2}^{2}}{4 R^{2}} .
$$

Using cylindrical coordinates for the point on the sphere

$$
x_{1}=r \cos \theta, \quad x_{2}=r \sin \theta, \quad x_{3}=x_{3},
$$

and polar coordinates on the plane passing through the south pole

$$
X_{1}=r_{1} \cos \Theta, \quad X_{2}=r_{1} \sin \Theta
$$

it turns out that

$$
r=\frac{r_{1}}{1+\frac{r_{1}^{2}}{4 R^{2}}}, \quad x_{3}=R \frac{-1+\frac{r_{1}^{2}}{4 R^{2}}}{1+\frac{r_{1}^{2}}{4 R^{2}}}, \quad \theta=\Theta .
$$

The expression for $r$ coincides with the one given in Eq. (15). We therefore see that the conformal factor can be seen as coming from a stereographic projection of a point on the surface of a $3 \mathrm{D}$ sphere onto a $2 \mathrm{D}$ plane tangent to it at its south pole. Such a stereographic projection is used in 6D in the framework of the IBM, in order to get the

classical IBM Hamiltonian in terms of the usual collective coordinates, on the 5D plane passing through the south pole of the relevant sphere [13].

\subsection{Embedding from five into six dimensions}

The usual Bohr Hamiltonian lives in an euclidean 5D space, the coordinates of which can be labelled as

$$
q_{1}=\Phi, \quad q_{2}=\Theta, \quad q_{3}=\psi, \quad q_{4}=\beta, \quad q_{5}=\gamma
$$

These coordinates play the role held by the polar coordinates in the $2 \mathrm{D}$ euclidean space, or by the spherical coordinates in the 3D euclidean space.

The line element is [5]

$$
d s^{2}=g_{i j} d q_{i} d q_{j}
$$

Using the explicit expressions for the matrix elements $g_{i j}$ given in [9], the line element takes the form

$$
d s^{2}=d \beta^{2}+\beta^{2} d \Omega_{4}^{2}
$$


where the hyperangular element is

$$
\begin{gathered}
d \Omega_{4}^{2}=4\left[\sin ^{2}\left(\gamma-\frac{2 \pi}{3}\right) \sin ^{2} \psi+\sin ^{2}\left(\gamma-\frac{4 \pi}{3}\right) \cos ^{2} \psi\right] d \Theta^{2} \\
+4\left[\sin ^{2}\left(\gamma-\frac{2 \pi}{3}\right) \sin ^{2} \Theta \cos ^{2} \psi+\sin ^{2}\left(\gamma-\frac{4 \pi}{3}\right) \sin ^{2} \Theta \sin ^{2} \psi+\sin ^{2} \gamma \cos ^{2} \Theta\right] d \Phi^{2} \\
+4 \sin ^{2} \gamma d \psi^{2}+8 \sin ^{2} \gamma \cos \Theta d \Phi d \psi+d \gamma^{2}-8 \sqrt{3} \sin \gamma \cos \gamma \sin \Theta \sin \psi \cos \psi d \Phi d \Theta
\end{gathered}
$$

Eq. (27) is the 5D analogue of Eq. (17) in 2D, and of Eq. (19) in 3D, i.e., the euclidean expression for the line element. $d \Omega_{4}^{2}$, which depends on the four angles $\Phi, \Theta, \psi, \gamma$, is the 5D analogue of $d \theta^{2}$ in 2D (which depends on the single angle $\theta$ ), and of $d \theta^{2}+\sin ^{2} \theta d \phi^{2}$ in 3D (which depends on the two angles $\theta, \phi$ ).

The form of Eq. (27) is in agreement with the fact that the configuration space $R_{5}$ of the Bohr model is known [30, 31] to be the tensor product of a radial line, $R_{+}$, and a four sphere, $S_{4}$.

Letting the nuclear mass $B$ to depend on the deformation $\beta$, it has been proved in [9] that the matrix elements become $g_{i j}^{\prime}=g_{i j} / f^{2}$, where $f(\beta)$ is the function expressing this dependence in Eq. (3). As a consequence, the line element of Eq. (26) becomes

$$
\left(d s^{\prime}\right)^{2}=g_{i j}^{\prime} d q_{i} d q_{j}
$$

leading to

$$
\left(d s^{\prime}\right)^{2}=\left(d \beta^{2}+\beta^{2} d \Omega_{4}^{2}\right) f^{-2}
$$

which is the analogue in 5D of Eqs. (16) in $2 \mathrm{D}$ and $(18)$ in $3 \mathrm{D}$.

In the case of the Davidson potential of Eq. (6), in which $f(\beta)$ is given by Eq. (7), the present Eq. (30) becomes

$$
\left(d s^{\prime}\right)^{2}=\left(d \beta^{2}+\beta^{2} d \Omega_{4}^{2}\right)\left(1+a \beta^{2}\right)^{-2} .
$$

This result is similar to Eqs. (16) and (18). This implies that $\beta$ has resulted from a conformal transformation

$$
\bar{\beta}=\beta\left(1+\frac{\beta^{2}}{4 R^{2}}\right)^{-1},
$$

with $a=1 /\left(4 R^{2}\right)$. 
Bohr Hamiltonian with a deformation-dependent mass term

Therefore we see that the DDM parameter $a$ is connected to the radius of curvature $R=1 /(2 \sqrt{a})$ of a hypersphere in $5 \mathrm{D}$, in agreement with the findings of [11]. The case of mass independent of the deformation, i.e., $a=0$, corresponds to a hypersphere in $5 \mathrm{D}$ of infinite radius, i.e. to a hyperplane in 5D. It should be remembered [13] at this point that the geometry of the interacting boson model (IBM) [7] is that of a 5D space. Therefore, the DDM Bohr results can be compared to the classical limit of the IBM, a task to be undertaken in the next section.

\section{The classical limit of the interacting boson model}

We now turn to the most general IBM Hamiltonian, which reads [7, 14]

$$
\begin{gathered}
H=\epsilon_{s} s^{\dagger} s+\epsilon_{d} d^{\dagger} \cdot \tilde{d}+u_{0}\left(s^{\dagger}\right)^{2} s^{2}+u_{2} s^{\dagger} d^{\dagger} \cdot \tilde{d} s+v_{0}\left[d^{\dagger} \cdot d^{\dagger} s^{2}+\left(s^{\dagger}\right)^{2} \tilde{d} \cdot \tilde{d}\right] \\
+v_{2}\left[\left(d^{\dagger} d^{\dagger}\right)^{(2)} \cdot \tilde{d} s+s^{\dagger} d^{\dagger} \cdot(\tilde{d} \tilde{d})^{(2)}\right]+\sum_{L=0,2,4} C_{L}\left(d^{\dagger} d^{\dagger}\right)^{(L)} \cdot(\tilde{d} \tilde{d})^{L)}
\end{gathered}
$$

where the symbols preceding the terms involving the boson creation $\left(s^{\dagger}, d^{\dagger}\right)$ and annihilation $\left(s, \tilde{d}=(-1)^{\mu} d_{-\mu}\right)$ operators are free parameters.

The corresponding Bohr Hamiltonian, obtained through the appropriate intrinsic state, reads [14]

$$
\begin{array}{r}
H_{B}=P_{1} \frac{\partial}{\partial \beta}+P_{2} \frac{\partial^{2}}{\partial \beta^{2}}+P_{12} \frac{\partial^{2}}{\partial \beta \partial \gamma}+\bar{P}_{1} \frac{1}{\beta} \frac{\partial}{\partial \gamma} \\
+\bar{P}_{2} \frac{1}{\beta^{2} \sin 3 \gamma} \frac{\partial}{\partial \gamma} \sin 3 \gamma \frac{\partial}{\partial \gamma}+{ }_{\sum^{-}}^{k=1,2,3} \frac{\hbar^{2} Q_{k}^{2}}{2 \mathcal{J}_{k}}+V_{N},
\end{array}
$$

with the explicit expressions of the coefficients appearing here given in terms of the coefficients of Eq. (33) and the total number of bosons $N$ in Ref. [14].

A detailed comparison of Eqs. (8) and (34), taking into account the rescaling which has been performed in Eq. (8), turns out to be instructive.

1) Since in Eq. (34) the coefficients contain the 9 parameters appearing in Eq. (33), while in relation to Eq. (8) only two parameters enter ( $a$, and the rescaling quantity $\left.\hbar^{2} / B_{0}\right)$, it is clear that any attempt to fully map Eq. (8) onto Eq. (34) will end up with an overcomplete set of equations among the 9 parameters of Eq. (33). 
Bohr Hamiltonian with a deformation-dependent mass term

2) No terms of the form $\partial / \partial \gamma$ and $\frac{\partial^{2}}{\partial \beta \partial \gamma}$ appear in 8 . Since the relevant coefficients in (34),

$$
\begin{aligned}
& \bar{P}_{1}=\sqrt{\frac{2}{7}} v_{2}\left(1+\frac{3 N \beta^{2}}{1+\beta^{2}}\right) \sin 3 \gamma, \\
& P_{12}=\sqrt{\frac{2}{7}} v_{2}\left(2-\beta^{2}\right) \sin 3 \gamma,
\end{aligned}
$$

are proportional to $\sin 3 \gamma[14$, it turns out that the DDM Hamiltonian (8) is missing some triaxiality effects contained in the classical limit of the most general IBM Hamiltonian (34). Since these coefficients are also proportional to $v_{2}$, we are going to ignore terms proportional to $v_{2}$ in what follows, formally considering $v_{2}=0$.

3) The coefficients of the term $(\sin 3 \gamma)^{-1}(\partial / \partial \gamma) \sin 3 \gamma(\partial / \partial \gamma)$ [14] lead to

$$
\bar{P}_{2}=v_{0}+\left(\frac{1}{5} C_{0}-\frac{2}{7} C_{2}+\frac{3}{35} C_{4}\right) \beta^{2}+\sqrt{\frac{2}{7}} v_{2} \beta \cos 3 \gamma=-\frac{\hbar^{2}}{2 B_{0}}\left(1+a \beta^{2}\right)^{2},
$$

from which the following remarks follow.

a) As above, there is a term $\beta \cos 3 \gamma$ in (34), arising from the $v_{2}$ term in (33). As discussed in 2), no such term exists in (8).

b) A $\beta^{4}$ term arises in (8), but not in 34. In other words, the DDM Bohr Hamiltonian contains some higher order terms not present in the classical limit of the IBM Hamiltonian. On the other hand, these higher order terms are preceded by $a^{2}$, which is a small quantity since $a<<1[9]$.

c) The coefficients of $\beta^{2}$ lead to

$$
\tilde{a}=-\frac{1}{5} C_{0}+\frac{2}{7} C_{2}-\frac{3}{35} C_{4}
$$

where

$$
\tilde{a}=\frac{\hbar^{2}}{B_{0}} a
$$

thus connecting the DDM parameter a to certain IBM parameters.

4) The coefficients of the terms containing the components of the angular momentum, $Q_{k}$,

$$
\frac{\hbar^{2}}{2 \mathcal{J}_{k}}=-\frac{1}{7}\left(C_{2}-C_{4}\right)-\frac{v_{0}+\left(\frac{1}{5} C_{0}-\frac{2}{7} C_{2}+\frac{3}{35} C_{4}\right) \beta^{2}+\sqrt{\frac{2}{7}} v_{2} \beta \cos \left(\gamma-\frac{2 \pi k}{3}\right)}{4 \beta^{2} \sin ^{2}\left(\gamma-\frac{2 \pi k}{3}\right)}
$$


lead to the same results as in 3$)$.

The results up to now can be summarized as follows.

1) The DDM Bohr Hamiltonian contains some higher order terms not present in the IBM Hamiltonian.

2) On the contrary, the classical limit of the IBM Hamiltonian contains $\gamma$-dependent terms absent from the DDM Bohr Hamiltonian.

3) It is interesting to see what are the implications of Eq. (38) in the three limiting symmetries of IBM.

Before proceeding to the study of the three limiting symmetries of the IBM, a comment on terms allowed in the general form of the Bohr collective model is in place. In general, terms of the form $\beta^{i}(\cos 3 \gamma)^{j}$ can occur [32. The term $i=0$ is always excluded, since for $j \neq 0$ it implies an indefinite value for the potential at $\beta=0$, while for $j=0$ it is just a constant [32, 33]. The well known 24 transformations which have to leave the wave function invariant [5, 34] imply that even values of $i$ should be accompanied by even values of $j$, or $j=0$, while odd values of $i$ should be accompanied by odd values of $j$. Usually, $i=1$ is not included in the potentials used in the collective model [33, 35, 36], since it does not have a smooth behaviour at $\beta=0$, but it could be included [33], since it does not violate any symmetry constraints. Therefore the appearance of the term $\beta \cos 3 \gamma$ in the above discussion is not problematic.

\subsection{The $U(5)$ limit}

In the $\mathrm{U}(5)$ (vibrational) limit a simple IBM Hamiltonian can be written as [7, 14]

$$
H_{\mathrm{U}(5)}=\epsilon_{d} d^{\dagger} \cdot \tilde{d}+\kappa_{5} d^{\dagger} \cdot d^{\dagger} \tilde{d} \cdot \tilde{d}
$$

In other words, the only non-vanishing coefficients in (33) are $\epsilon_{d}$ and $C_{0}=5 \kappa_{5}$. Then Eq. (38) yields

$$
\tilde{a}=-\kappa_{5} \text {. }
$$

Thus in the vibrational limit the DDM parameter $a$ turns out to be related to the strength of the pairing interaction among the $d$-bosons. It should be pointed out that 
$\kappa_{5}$ obtains negative values (see Eqs. (4.11a) and (4.12) of Ref. [14]), thus guaranteeing that $a>0$, as it should be $[9]$.

A more general IBM Hamiltonian in the $\mathrm{U}(5)$ limit reads [7, 37]

$$
H_{\mathrm{U}(5)}=\epsilon_{d} d^{\dagger} \cdot \tilde{d}+\sum_{L=0,2,4} C_{L}\left(d^{\dagger} d^{\dagger}\right)^{(L)} \cdot(\tilde{d} \tilde{d})^{(L)}
$$

in which the non-vanishing coefficients in (33) are $\epsilon_{d}, C_{0}, C_{2}, C_{4}$. In this case, $\tilde{a}$ is given by Eq. (38).

\subsection{The $S U(3)$ limit}

We assume now a quadrupole-quadrupole Hamiltonian of the form [7, 38]

$$
H_{\mathrm{SU}(3)}=-\kappa Q \cdot Q-\kappa^{\prime} L \cdot L
$$

where $Q$ is the quadrupole operator

$$
Q=s^{\dagger} \tilde{d}_{\mu}+d_{\mu}^{\dagger} s+\chi\left(d^{\dagger} \tilde{d}\right)_{\mu}^{(2)}
$$

and $L$ is the angular momentum operator

$$
L=\sqrt{10}\left(d^{\dagger} \tilde{d}\right)^{(1)}
$$

This choice of the Hamiltonian corresponds to specific values of the parameters of Eq. (33) in terms of $\kappa$ and $\kappa^{\prime}$, including [38, 14]

$$
C_{0}=-\frac{7}{4} \kappa+6 \kappa^{\prime}, \quad C_{2}=\frac{3}{8} \kappa+3 \kappa^{\prime}, \quad C_{4}=-\frac{1}{2} \kappa-4 \kappa^{\prime},
$$

in which the differences in the definitions of the parameters in Table I of Ref. 38] and in Eq. (5.19) of Ref. [14] have been taken into account, as shown in the Appendix A1.1. These parameters, used in Eq. (38), lead to

$$
\tilde{a}=\frac{1}{2} \kappa
$$

Thus in the SU(3) limit the DDM parameter $a$ turns out to be related to the strength of the quadrupole-quadrupole interaction, while the coefficient of the angular momentum term does not enter. It is known [38] that $\kappa$ takes positive values, thus guaranteeing $a>0$, as it should be $[9$. 


\subsection{The $O(6)$ limit}

In the $\mathrm{O}(6)$ ( $\gamma$-unstable) limit the most general Hamiltonian takes the form [7, 39]

$$
H_{\mathrm{O}(6)}=A P_{6}+B C_{5}+C L \cdot L
$$

where $L$ is given by Eq. 46, and

$$
P_{6}=P^{\dagger} \cdot \tilde{P}, \quad \tilde{P}=\frac{1}{2}(\tilde{d} \cdot \tilde{d})-\frac{1}{2}(\tilde{s} \cdot \tilde{s}), \quad C_{5}=\frac{1}{3} \sum_{1,3}\left(d^{\dagger} \tilde{d}\right)^{(k)} \cdot\left(d^{\dagger} \tilde{d}\right)^{(k)} \cdot(50)
$$

This choice of the Hamiltonian corresponds to specific values of the parameters of Eq. (33) in terms of $A, B, C$, including [39]

$$
C_{0}=\frac{5}{4} A-\frac{2}{3} B-6 C, \quad C_{2}=\frac{1}{6} B-3 C, \quad C_{4}=\frac{1}{6} B+4 C,
$$

in which the differences in the definitions of the parameters in Table I of Ref. [39] and in Ref. [14] have been taken into account, as shown in the Appendix A1.2 . These parameters, used in Eq. (38), lead to

$$
\tilde{a}=-\frac{1}{4} A+\frac{1}{6} B
$$

Thus in the $\mathrm{O}(6)$ limit the DDM parameter $a$ turns out to be related to the difference of the strengths of a pairing interaction involving both $s$ and $d$ bosons and a pairing interaction involving $d$ bosons only, while the coefficient of the angular momentum term again does not enter.

\subsection{Discussion}

We have found that in all the $\mathrm{U}(5)$, $\mathrm{SU}(3)$, and $\mathrm{O}(6)$ limits the DDM parameter $a$ is connected to relevant IBM parameters.

It should be remarked that the very presence of non-vanishing $d$-boson pairing interaction in vibrational nuclei, or of non-vanishing quadrupole-quadrupole interaction in rotational nuclei, or of non-vanishing difference between the pairing interactions among $s, d$ bosons and $d$ bosons alone in $\gamma$-unstable nuclei, requires a non-vanishing value of the DDM parameter $a$, implying a finite curvature of the 5D Bohr space. In other words, the classical limit of the IBM Hamiltonian corresponds to a curved 5D 
space [12], while in the original Bohr Hamiltonian the 5D space is flat (corresponding to $a=0$, i.e., to infinite radius of curvature).

The connection between curvature and interaction appears in several branches of physics.

1) In the general theory of relativity [40], the Einstein field equation (actually a set of 10 equations) connects the local spacetime curvature (expressed by the Einstein tensor, constructed from the Riemannian curvature tensor and the metric) to the local energy and momentum within that spacetime (expressed by the stress-energy tensor, defined by the matter content of the spacetime), thus describing quantitatively the principle that "spacetime tells matter how to move, and matter tells spacetime how to curve" 41].

2) In thermodynamics, the Ruppeiner geometry has been developed [42, 43], in which thermodynamical systems are represented in terms of Riemannian geometry, the relevant metric being flat for noninteracting particles, while curvature develops in the presence of interactions [44].

Connections between curvature and interaction have also been considered in the realm of nuclear structure.

1) In the search for a collective path in the many-particle Hilbert space, the manifold of Slater determinants has been considered as a Riemannian manifold [45], with the curvature of a collective path, expressed through an external curvature tensor, found to be related to its collectivity. In particular, geodesics (lines of zero internal curvature) on generic $\mathrm{SU}(3)$ orbits are found to be highly collective paths [45].

2) By interpreting geometrically the collective masses, the metric tensor can be defined, which fully determines the geometric properties of the collective Riemannian space [46]. Assuming non-vanishing curvature in collective space, the finite range liquid drop model with curvature, and the finite range droplet model with curvature, have been constructed, giving improved ground state properties for light nuclei and trans-fermium elements [46]. 
In view of the above comments, the present results can be interpreted in the following way. The curvature of the space is determined by the leading interaction present in each symmetry limit of the IBM. Since the curvature is directly related to the free parameter $a$ appearing in the dependence of the mass on the deformation, it turns out that the parameter $a$ is also determined by the leading interaction present in each symmetry limit.

\section{Conclusions}

The physical meaning of the free parameter $a$ appearing in the dependence of the mass on the deformation in the Bohr Hamiltonian with a Davidson potential has been considered. The main results are summarized here.

1) By embedding the 5D DDM Bohr space into a 6D space, the parameter $a$ has been connected to the curvature of the 5D space, the original Bohr Hamiltonian corresponding to a flat $5 \mathrm{D}$ space.

2) By comparing the deformation-dependent mass (DDM) Bohr Hamiltonian to the classical limit of the most general IBM Hamiltonian, the parameter $a$ has been connected to certain IBM parameters. In particular the parameter $a$ has been found a) in the $\mathrm{U}(5)$ limit to be proportional to the strength of the $d$-boson pairing interaction, b) in the $\mathrm{SU}(3)$ limit to be proportional to the strength of the quadrupole-quadrupole interaction, c) in the $\mathrm{O}(6)$ limit to be proportional to a difference of the strengths of the pairing interaction among $s$ and $d$ bosons and the pairing interaction among $d$-bosons alone.

3) The presence of certain basic interactions in the IBM results in a curved 5D space corresponding to its classical limit, while the 5D space of the original Bohr Hamiltonian is a flat one. Curvature needs to be added to the 5D space of the Bohr Hamiltonian, by allowing the nuclear mass to depend on the deformation, in order to establish agreement with the classical limit of IBM. In other words, the IBM in its classical limit, as already remarked in Ref. [14], has built-in the dependence of the nuclear mass 
on the deformation, which has been introduced in the DDM Bohr Hamiltonian in order to fix the behaviour of the moments of inertia as functions of the deformation.

The influence of the parameter $a$, i.e. of the curvature of the 5D space, on the properties of critical point symmetries [47, 48] and shape phase transitions [49, 50] in atomic nuclei is an interesting problem to be pursued. The embedding of the Bohr space in $6 \mathrm{D}$ has already been used in revealing the $\mathrm{O}(6)$ symmetry and its contraction to the $\mathrm{E}(5)$ symmetry at infinity [11].

The DDM approach to the Bohr Hamiltonian with a Kratzer potential [26] has been recently carried out [27, providing a different factor $f(\beta)=1+\alpha \beta$ (with $a<<1$ ). The extension of the present embedding approach to the Kratzer case is an interesting task, which might require the use of a different coherent state.

\section{Acknowledgements}

The authors are thankful to P. E. Georgoudis for useful discussions. Financial support from the Bulgarian National Science Fund under contract No. DFNI-E02/6 and by the Scientific Research Projects Coordination Unit of Istanbul University under Project No 50822 is gratefully acknowledged.

\section{Appendix: Coefficients of different Hamiltonians}

In the most general IBM Hamiltonian given in Eq. (33) as written in Ref. [14], there are some differences in the coefficients in comparison to the most general IBM Hamiltonian reported in Ref. [7]

$$
\begin{gathered}
H=\epsilon_{s} s^{\dagger} s+\epsilon_{d} d^{\dagger} \cdot \tilde{d}+\frac{1}{2} u_{0}\left(s^{\dagger}\right)^{2} s^{2}+u_{2}\left[\left(s^{\dagger} d^{\dagger}\right)^{(2)} \times(\tilde{d} s)^{(2)}\right]^{(0)} \\
+\frac{1}{2} v_{0}\left[\left(d^{\dagger} d^{\dagger}\right)^{(0)} \times(s s)^{(0)}+\left(s^{\dagger} s^{\dagger}\right)^{(0)} \times(d \tilde{d})^{(0)}\right]^{(0)} \\
+v_{2}\left[\left(d^{\dagger} d^{\dagger}\right)^{(2)} \times(\tilde{d} s)^{(2)}+\left(s^{\dagger} d^{\dagger}\right)^{(2)} \times(\tilde{d} \tilde{d})^{(2)}\right]^{(0)} \\
+\sum_{L=0,2,4} \frac{1}{2} \sqrt{2 L+1} C_{L}\left[\left(d^{\dagger} d^{\dagger}\right)^{(L)} \times(\tilde{d} \tilde{d})^{L}\right]^{(0)}
\end{gathered}
$$


Bohr Hamiltonian with a deformation-dependent mass term

Using the identity

$$
U^{(k)} \cdot V^{(k)}=(-1)^{k} \sqrt{2 k+1}\left(U^{(k)} V^{(k)}\right)^{(0)},
$$

one easily finds the following relations between the Iachello-Arima parameters of Eq. (53), labelled by IA, and the Ginocchio-Kirson parameters of Eq. (33), labelled by GK

$$
\begin{gathered}
\epsilon_{s, G K}=\epsilon_{s, I A}, \quad \epsilon_{d, G K}=\epsilon_{d, I A}, \quad \sqrt{5} v_{0, G K}=\frac{1}{2} v_{0, I A}, \\
\sqrt{5} v_{2, G K}=\frac{1}{\sqrt{2}} v_{2, I A}, \quad u_{0, G K}=\frac{1}{2} u_{0, I A}, \quad \sqrt{5} u_{2, G K}=u_{2, I A}, \\
C_{0, G K}=\frac{1}{2} C_{0, I A}, \quad C_{2, G K}=\frac{1}{2} C_{2, I A}, \quad C_{4, G K}=\frac{1}{2} C_{4, I A} .
\end{gathered}
$$

\section{$A 1.1 S U(3)$}

From Table I of Ref. 38 one reads

$$
\begin{aligned}
\epsilon_{s, I A}=-5 \kappa, \epsilon_{d, I A}=-\frac{11}{4} \kappa-6 \kappa^{\prime}, \quad v_{0, I A}=-2 \sqrt{5} \kappa, \quad v_{2, I A}=\sqrt{70} \kappa, & u_{0, I A}=0 \\
u_{2, I A}=-2 \sqrt{5} \kappa, \quad C_{0, I A}=-\frac{7}{2} \kappa+12 \kappa^{\prime}, \quad C_{2, I A}=\frac{3}{4} \kappa+6 \kappa^{\prime}, & C_{4, I A}=-\kappa-8 \kappa^{\prime} .(56
\end{aligned}
$$

Taking into account the relations of Eq. (55) one finds

$$
\begin{gathered}
\epsilon_{s, G K}=-5 \kappa, \epsilon_{d, G K}=-\frac{11}{4} \kappa-6 \kappa^{\prime}, \quad v_{0, G K}=-\kappa, \quad v_{2, G K}=\sqrt{7} \kappa, \quad u_{0, G K}=0 \\
u_{2, G K}=-2 \kappa, \quad C_{0, G K}=-\frac{7}{4} \kappa+6 \kappa^{\prime}, \quad C_{2, G K}=\frac{3}{8} \kappa+3 \kappa^{\prime}, \quad C_{4, G K}=-\frac{1}{2} \kappa-4 \kappa^{\prime},
\end{gathered}
$$

in agreement to Eq. (5.19) of Ref. [14] as far as the $\kappa$ terms are concerned.

\section{A1.2 $O(6)$}

From Table I of Ref. [39] one reads

$$
\begin{gathered}
\epsilon_{s, I A}=0, \epsilon_{d, I A}=\frac{2}{3} B+6 C, \quad v_{0, I A}=-\frac{\sqrt{5}}{2} A, \quad v_{2, I A}=0, \quad u_{0, I A}=\frac{1}{2} A, \\
u_{2, I A}=0, \quad C_{0, I A}=\frac{5}{2} A-\frac{4}{3} B-12 C, \quad C_{2, I A}=\frac{1}{3} B-6 C, \quad C_{4, I A}=\frac{1}{3} B+8 C .
\end{gathered}
$$

Taking into account the relations of Eq. 55 one finds

$$
\begin{gathered}
\epsilon_{s, G K}=0, \epsilon_{d, G K}=\frac{2}{3} B+6 C, \quad v_{0, G K}=-\frac{1}{4} A, \quad v_{2, G K}=0, \quad u_{0, G K}=\frac{1}{4} A, \\
u_{2, G K}=0, \quad C_{0, G K}=\frac{5}{4} A-\frac{2}{3} B-6 C, \quad C_{2, G K}=\frac{1}{6} B-3 C, \quad C_{4, G K}=\frac{1}{6} B+4 C .(59)
\end{gathered}
$$




\section{References}

[1] Cooper F, Khare A and Sukhatme U 1995 Supersymmetry and quantum mechanics Phys. Rep. $251267-385$

[2] Cooper F, Khare A and Sukhatme U 2001 Supersymmetry in Quantum Mechanics (Singapore: World Scientific)

[3] Quesne C and Tkachuk V M 2004 Deformed algebras, position-dependent effective masses and curved spaces: an exactly solvable Coulomb problem J. Phys. A: Math. Gen. 37 4267-4281

[4] Bagchi B, Banerjee B A, Quesne C and Tkachuk V M 2005 Deformed shape invariance and exactly solvable Hamiltonians with position-dependent effective mass J. Phys. A: Math.Gen. 38 29292945

[5] Bohr A 1952 The coupling of nuclear surface oscillations to the motion of individual nucleons Mat. Fys. Medd. K. Dan. Vidensk. Selsk. 26 no. 14

[6] Bohr A and Mottelson B R 1975 Nuclear Structure Vol. II: Nuclear Deformations (New York: Benjamin)

[7] Iachello F and Arima A 1987 The Interacting Boson Model (Cambridge: Cambridge University Press)

[8] Ring P and Schuck P 1980 The Nuclear Many-Body Problem (Berlin: Springer)

[9] Bonatsos D, Georgoudis P E, Lenis D, Minkov M and Quesne C 2011 Bohr Hamiltonian with a deformation-dependent mass term for the Davidson potential Phys. Rev. C $\mathbf{8 3} 044321$

[10] Davidson P M 1932 Eigenfunctions for calculating electronic vibrational intensities Proc. R. Soc. London Ser. A 135 459-472

[11] Georgoudis P E 2014 Bohr space in six dimensions Phys. Lett. B 731 122-125

[12] Ginocchio J N and Kirson M W 1980 Relationship between the Bohr collective Hamiltonian and the Interacting Boson Model Phys. Rev. Lett. 44 1744-1747

[13] Dieperink A E L, Scholten O and Iachello F 1980 Classical limit of the Interacting-Boson Model Phys. Rev. Lett. 44 1747-1750

[14] Ginocchio J N and Kirson M W 1980 An intrinsic state for the Interacting Boson Model and its relationship to the Bohr-Mottelson model Nucl. Phys. A 350 31-60

[15] van Roosmalen O S 1982 Algebraic descriptions of nuclear and molecular rotation-vibration spectra Ph.D. thesis, U. Groningen

[16] Jolos R V and von Brentano P 2007 Bohr Hamiltonian with different mass coefficients for the ground- and $\gamma$ bands from experimental data Phys. Rev. C 76, 024309

[17] Jolos R V and von Brentano P 2009 Mass tensor in the Bohr Hamiltonian from the nondiagonal energy weighted sum rules Phys. Rev. C $\mathbf{7 9} 044310$ 
[18] Jolos R V and von Brentano P $2009 \mathrm{X}(5)^{*}$ model and the $N=90$ nuclei Phys. Rev. C 80034308

[19] Ermamatov M J and Fraser P R 2011 Rotational-vibrational excited states of axially symmetric nuclei with different mass parameters Phys. Rev. C 84044321

[20] Ermamatov M J, Srivastava P C, Fraser P R, Stránsky P and Morales I O 2012 Coriolis contribution to excited states of deformed ${ }^{163} \mathrm{Dy}$ and ${ }^{173} \mathrm{Yb}$ nuclei with multiple mass parameters Phys. Rev. $C 85034307$

[21] Rowe D J 2004 A computationally tractable version of the collective model Nucl. Phys. A 735 372-392

[22] Rowe D J and Turner P S 2005 The algebraic collective model Nucl. Phys. A 753 94-105

[23] Rowe D J, Welsh T A and Caprio M A 2009 Bohr model as an algebraic collective model Phys. Rev. C 79054304

[24] Wilets L and Jean M 1956 Surface oscillations in even-even nuclei Phys. Rev. 102 788-796

[25] Fortunato L 2005 Solutions of the Bohr Hamiltonian, a compendium Eur. Phys. J. A 26 s01, 1-30

[26] Kratzer A 1920 Eine ultraroten Rotationsspektren der Halogenwasserstoffe Z. Physik 3 289-307

[27] Bonatsos D, Georgoudis P E, Minkov N, Petrellis D and Quesne C 2013 Bohr Hamiltonian with a deformation-dependent mass term for the Kratzer potential Phys. Rev.C $\mathbf{8 8} 034316$

[28] Robertson H P 1933 Relativistic cosmology Rev. Mod. Phys. 5 62-90

[29] Landau L D and Lifthitz E M 1975 The classical theory of fields (Oxford: Pergamon)

[30] Rowe D J 2005 An algebraic approach to problems with polynomial Hamiltonians on Euclidean spaces J. Phys. A: Math. Gen. 38 10181-10201

[31] Rowe D J and Wood J L 2010 Fundamentals of Nuclear Models (Singapore: World Scientific)

[32] Greiner W and Maruhn J A 1996 Nuclear Models (Berlin: Springer)

[33] Hess P O, Seiwert M, Maruhn J and Greiner W 1980 General collective model and its application to ${ }_{92}^{238} \mathrm{U}$ Z. Phys. A: Atoms and Nuclei 296 147-163

[34] Corrigan T M, Margetan F J and Williams S A 1976 Exact solution of the quadrupole surface vibration Hamiltonian in body-fixed coordinates Phys. Rev. C 14 2279-2296

[35] Hess P O, Maruhn J and Greiner W 1981 The general collective model applied to the chains of Pt, Os and W isotopes J. Phys. G: Nucl. Phys. 7 737-769

[36] Gneuss G and Greiner W 1971 Collective potential energy surfaces and nuclear structure Nucl. Phys. A 171 449-479

[37] Arima A and Iachello F 1976 Interacting Boson Model of collective states I. The vibrational limit Ann. Phys. (NY) 99 253-317

[38] Arima A and Iachello F 1978 Interacting Boson Model of collective nuclear states II. The rotational limit Ann. Phys. (NY) 111 201-238 
[39] Arima A and Iachello F 1979 Interacting Boson Model of collective nuclear states IV. The O(6) limit Ann. Phys. (NY) 123 468-492

[40] Einstein A 1916 Die Grundlage der allgemeinen Relativitätstheorie Annalen der Physik 49 769-822

[41] Wheeler J A 1990 A Journey into Gravity and Spacetime (San Francisco: Scientific American Library)

[42] Ruppeiner G 1995 Riemannian geometry in thermodynamic fluctuation theory Rev. Mod. Phys. $67605-659$

[43] Ruppeiner G 2010 Thermodynamic curvature measures interactions Am. J. Phys. 78 1170-1180

[44] Quevedo H, Sánchez A, Taj S and Vázquez A 2010 Curvature as a measure of the thermodynamic interaction J. Korean Phys. Soc. 57 646-650

[45] Giraud B G and Rowe D J 1979 Curvature as a measure of collectivity Nucl. Phys. A 330 352-366

[46] Herrmann R 2012 Curvature interaction in collective space Int. J. Mod. Phys. E 211250103

[47] Iachello F 2000 Dynamic symmetries at the critical point Phys. Rev. Lett. 85 3580-3583

[48] Iachello F 2001 Analytic description of critical point nuclei in a spherical-axially deformed shape phase transition Phys. Rev. Lett. 87052502

[49] Casten R F and McCutchan E A 2007 Quantum phase transitions and structural evolution in nuclei J. Phys. G: Nucl. Part. Phys. 34 R285-R320

[50] Cejnar P, Jolie J and Casten R F 2010 Quantum phase transitions in the shapes of atomic nuclei Rev. Mod. Phys. 82 2155-2212 